\title{
The Nix locus on the male-specific homologue of chromosome 1 in Aedes albopictus is a strong candidate for a male- determining factor
}

\author{
Ludvik M. Gomulski ${ }^{1}$, Marina Mariconti ${ }^{1}$, Alessandro Di Cosimo ${ }^{1}$, Francesca Scolari ${ }^{1}$, Mosè Manni ${ }^{1}{ }^{2}$, Grazia Savini ${ }^{1}$, \\ Anna R. Malacrida ${ }^{1}$ and Giuliano Gasperi ${ }^{{ }^{*}}$
}

\begin{abstract}
Background: Global concern over the rapid expansion of the Asian tiger mosquito, Aedes albopictus, and its vector competence has highlighted an urgent need to improve currently available population control methods, like the Sterile Insect Technique. Knowledge of the sex determination cascade is a prerequisite for the development of early-stage sexing systems. To this end, we have characterised the putative sex determination gene, Nix, in this species. In Aedes species the chromosome complement consists of three pairs of chromosomes. The sex determination alleles are linked to the smallest homomorphic chromosome.

Results: We identified the male-specific chromosome 1 of Ae. albopictus that carries the putative male-determining gene Nix. We have also characterised the complete genomic sequence of the Nix gene which is composed of two exons and a short intron. The gene displays different levels of intron retention during development. Comparison of DNA sequences covering most of the Nix gene from individuals across the species range revealed no polymorphism.

Conclusions: Our characterisation of the Nix gene in Ae. albopictus represents an initial step in the analysis of the sex determination cascade in this species. We found evidence of intron retention (IR) in Nix. IR might play a role in regulating the expression of Nix during development. Our results provide the basis for the development of new genetic control strategies.
\end{abstract}

Keywords: Nix, sex determination, sex chromosome, intron retention, virus vector, control strategies

\section{Background}

The Asian tiger mosquito, Aedes albopictus, is among the most aggressive and invasive mosquitoes in the world. It is a vector of numerous pathogens including the world's most prevalent arboviruses, dengue, chikungunya and Zika [1, 2]. Aedes albopictus has rapidly expanded from Southeast Asia to colonize both tropical and temperate countries and is now present in all continents [3-5] and has caused major chikungunya outbreaks in parts of Europe including Italy $[6,7]$ and France [8].

\footnotetext{
*Correspondence: gasperi@unipv.it

'Department of Biology and Biotechnology "L. Spallanzani", University of Pavia, Pavia, Italy

Full list of author information is available at the end of the article
}

The global concern over the rapid expansion and the ability of Ae. albopictus to transmit infectious diseases has highlighted an urgent need to improve currently available population control methods [9]. These include the Sterile Insect Technique, which requires highly efficient early-stage sexing methods [10]. A better understanding of the sex determination mechanisms in $A e$. albopictus would be thus of practical importance and would also benefit evolutionary studies of sex-specific sequences.

Unlike anophelines, the sex chromosomes are homomorphic in all culicine mosquitoes including Aedes species. In these species the chromosome complement consists of three pairs of chromosomes designated 1, 2 and 3 [11]. The sex determination alleles are linked to 
the smallest homomorphic chromosome 1 and are described as $\mathrm{Mm}$ in males and $\mathrm{mm}$ in females [12-14]. The homomorphic sex chromosome arrangement is considered the ancestral state of mosquito sex chromosomes [15]. Differences between the male and female sex chromosomes in the mitotic complements of nine Aedes species have been studied using Giemsa C-banding [16]. From the C-banding patterns, it was suggested that two separate lines of evolution for the chromosome 1 homologues have taken place, one leading to Ae. aegypti type and the other to the Ae. albopictus type [16]. However, as the C-banding pattern has been found to be polymorphic in different Ae. aegypti strains, clear sex chromosome differences were not well defined. In Ae. aegypti the development of integrated linkage, chromosome and genomic maps [17] were the premise for the identification of the homomorphic chromosome $1 \mathrm{M}$ homologue and the location of a dominant male determining factor Nix in the M locus [18].

In Ae. aegypti the sex-determination locus resides in band $1 \mathrm{q} 21$ on the $\mathrm{q}$-arm of chromosome 1, a region that is not subject to recombination [19]. Beyond this region recombination is thought to occur in an autosome-like fashion, maintaining the overall homomorphic structure of this chromosome [19]. Further genetic evidence has recently shown that chromosome 1 in Ae. aegypti is differentiated over a region much larger than the M-locus and shows features of an XY chromosomal system despite the apparent homomorphy [20].

A number of male-specific genomic sequences were identified in Ae. aegypti using the chromosome quotient method [18]. Among these sequences was a novel gene, Nix, that shared moderate identity with transformer-2, one of the key sex-determination genes in Drosophila melanogaster [21]. The Ae. aegypti Nix gene is $985 \mathrm{bp}$ in length and encodes a 288 amino acid polypeptide with two RNA recognition motifs. Nix exhibits persistent male linkage and is expressed early in embryonic development [18]. These are essential characteristics of an $\mathrm{M}$ factor. Furthermore, somatic knockout of $\mathrm{Nix}$ in $\mathrm{Ae}$. aegypti male embryos results in feminization, and ectopic expression of Nix in female embryos results in masculinization, clearly demonstrating that Nix is sufficient for the initiation of male development [18]. Nix is hypothesised to be a splicing factor that acts directly or indirectly on the doublesex ( $d s x)$ and fruitless (fru) genes involved in sex-determination [22].

In Ae. albopictus the $\mathrm{M}$ and $\mathrm{m}$ homomorphic homologues of chromosome 1 have not been identified. Hall et al [18] identified an incomplete Nix homologue in Ae. albopictus (GenBank accession number KP765684). Recently, the complete Ae. albopictus Nix sequence was identified from a C6/36 cell line genome [23]. This complete sequence is almost identical to the Nix sequence that we independently identified in the $A e$. albopictus Rimini strain and that we describe both cytologically and functionally in this paper.

Using morphological, cytogenetics and molecular approaches, we have identified the male-specific chromosome 1, that carries the putative male-determining gene, Nix. This gene has all the characteristics of a dominant, male-determining factor. Characterisation of Nix in $\mathrm{Ae}$. albopictus revealed a possible mechanism (intron retention, IR) that regulates its expression during embryogenesis and development. Our findings may be applied to further understanding of the sex-determination cascade and the development of novel genetic control strategies that can help combat this important disease vector by converting females into harmless males. Furthermore, we have developed markers useful for sexing pre-imaginal Ae. albopictus individuals from the embryo to the pupal stage.

\section{Methods \\ Mosquitoes}

The Rimini laboratory strain was used. This strain was established in 2004 from eggs collected in ovitraps from Rimini, Emilia Romagna, Italy $\left(44^{\circ} 03^{\prime} 24^{\prime \prime} \mathrm{N}, 12^{\circ} 33^{\prime} 52^{\prime \prime} \mathrm{E}\right)$ [24]. The strain has been maintained in Pavia since its arrival in December 2013 at generation 53 from Romeo Bellini of the Centro Agricoltura Ambiente "G. Nicoli" in Crevalcore (Italy). The strain is maintained at $26{ }^{\circ} \mathrm{C}$ with $70 \%$ relative humidity and a 12:12 h (light:dark) photoperiod. Larvae are reared on fish food pellets (Tetra Goldfish Granules), whereas adults are maintained on $20 \%$ sugar solution and commercially available pig or cow blood using a membrane feeding apparatus.

Samples collected from wild populations were also used. These included individuals from northern Italy (Arco in Trento, collected in August 2012), Croatia (Velika Gorica, collected in August 2017), Réunion (St Denis, collected in March 2010), China (Guangzhou, collected in November 2017) and two from Thailand (Ban Rai and Chiang Mai, both collected in November 2010). The Thai Chiang Mai sample consisted of DNA extracted from a pool of individuals.

Pupae were sexed using the sexual dimorphism of the tenth abdominal segment or genital pouch [25].

\section{Isolation of developing gonads in Ae. albopictus larvae}

Fourth-instar larvae were dissected to isolate the developing gonads following the protocol described for $A e$. aegypti with modifications [26]. Briefly, individual fourth-instar larvae were transferred to a drop of phosphate-buffered saline (PBS) on a glass slide and the head removed. Using fine-tipped forceps, the abdomen was laterally incised at the level of the sixth segment and the cuticle removed to expose the gonads. The gonads 
were then transferred in a drop of PBS on clean glass slide, covered with a coverslip, and observed under a Zeiss Axioplan microscope at 200x and 400× magnification. Images were captured using an Olympus DP70 digital camera.

\section{Nucleic acid preparation and sequence analyses}

For large individuals, such as larvae, pupae and adults, the specimen was divided in two parts transversally and the abdomen used for RNA extraction using TRIzol Reagent (Invitrogen) following the manufacturer's protocol. The head and thorax were used for DNA extraction [27]. Likewise, for large egg collections, the sample was divided into two parts for DNA and RNA extraction. RNA was also extracted from different body compartments of 2-4 day-old males using TRIzol using the following pools: antennae ( 100 pairs), palps ( $\sim 100$ pairs), proboscises (25), heads without antennae, palps and proboscises (5), legs $(\sim 10$ sets), thoraces without wings and legs (5), abdomens (5) and wings (50 pairs). RNAs were treated with DNAse I (DNAfree, Ambion), whereas DNAs were treated with RNase A (Invitrogen). The nucleic acids were resuspended in DEPC-treated TE buffer $(10 \mathrm{mM}$ Tris- $\mathrm{HCl}, \mathrm{pH} 8,1$ mM EDTA) and concentrations were determined using a Nanodrop ND-1000 spectrophotometer (Nano-drop Technologies Inc, Wilmington, DE, USA).

PCR amplifications were performed using Taq DNA polymerase or AccuPrime Taq DNA Polymerase High Fidelity Kit (Invitrogen) using the following cycle conditions: $95{ }^{\circ} \mathrm{C}$ for $2 \mathrm{~min}, 35$ cycles at $95{ }^{\circ} \mathrm{C}$ for $20 \mathrm{~s}, 58{ }^{\circ} \mathrm{C}$ for $20 \mathrm{~s}, 72{ }^{\circ} \mathrm{C}$ for $1 \mathrm{~min}$, and a final extension at $72{ }^{\circ} \mathrm{C}$ for $5 \mathrm{~min}$ for the Nix-309, Nix-833 and 18S rDNA primers (Additional file 1: Table S1). The extension time was increased to $1 \mathrm{~min} 30 \mathrm{~s}$ for the Nix-1121 primer pair. The $18 \mathrm{~S}$ rDNA fragment was amplified as a control for DNA integrity. Amplification products were electrophoresed on $1.5 \%$ agarose gels and sequenced directly or cloned using the TOPO TA cloning kit (Invitrogen) before sequencing both strands (Macrogen Europe).

Synthesis of cDNA was performed using $200 \mathrm{ng}$ RNA (with the exception of single embryos where the complete RNA sample was used) in $20 \mu$ reaction volumes using the iScript ${ }^{\mathrm{TM}}$ cDNA Synthesis Kit (Bio-Rad). RT-PCRs with gene-specific primers, designed using Primer3Plus [28] (Additional file 1 : Table S1), were performed using $5 \%$ of the synthesized cDNA and the cycle conditions were those used for DNA amplifications. An $18 \mathrm{~S}$ rDNA fragment was amplified as a control for cDNA integrity. To check for genomic DNA contamination, RT-PCR was also performed on controls, i.e. RNA samples in which cDNA synthesis had been performed in the absence of reverse transcriptase. Amplification products were electrophoresed on $1.5 \%$ agarose gels. The amplification products were cloned and sequenced as above.

Sequence analyses were performed using the BLAST family of programs [29, 30] and Conserved Domain search [31] from the National Centre for Biotechnology Information. Sequences were aligned manually or using CLC Main Workbench 6.9.1 (CLC bio).

\section{Metaphase chromosome preparation and in situ hybridization}

Mitotic chromosome spreads were obtained from the imaginal discs of sexed fourth-instar larvae (Rimini strain) following the protocol of Sharakhova et al. [32]. Briefly, for each larva, the thorax, previously isolated in PBS, was transferred to cold hypotonic solution (1\% sodium citrate) and dissected to extract the imaginal discs. After incubation in $1 \%$ sodium citrate for $10 \mathrm{~min}$ at room temperature the imaginal discs were transferred to methanol-acetic acid 3:1 solution for $4 \mathrm{~min}$. Subsequently, $100 \mu \mathrm{l} 60 \%$ acetic acid was added to the material for chromosome fixation and the disrupted imaginal discs were dropped onto clean slides and dried. The slides were stained with DAPI (4',6-Diamidine-2' -phenylindole dihydrochloride; $10 \mathrm{ng} / \mathrm{ml}$ in $4 \times \mathrm{xSC}$ ) which produces a pattern similar to Hoechst 33258, which in Drosophila stains heterochromatic regions [33].

Fluorescence in situ hybridizations of Nix and 18S rDNA probes were performed on mitotic chromosome preparations obtained from sexed larvae. An 833 bp Nix sequence cloned in $\mathrm{pCR}^{\mathrm{TM}}$ 2.1-Topo vector, and a 757 bp $18 \mathrm{~S}$ rDNA PCR fragment were used as probes. The probes were labelled using the Biotin High Prime kit (Roche) and detection of hybridization signals was performed using the Alexa Fluor 594 Tyramide Signal Amplification Kit (Invitrogen). Chromosomes were counterstained and mounted using the VECTASHIELD mounting medium (Vector Laboratories, Burlingame, CA, USA). Hybridization and DAPI fluorescence signals were visualized through appropriate filters using a Zeiss Axioplan microscope. Images were captured using an Olympus DP70 digital camera with exposure times of 0.5 and $0.2 \mathrm{~s}$ for rhodamine and DAPI, respectively.

The karyotype description [34] was adopted for the chromosomal localization of Nix and 18S rDNA on mitotic chromosomes.

\section{Results}

\section{Sexing of fourth-instar larvae through dissection of developing gonads}

Larvae were sexed in order to obtain sex-specific mitotic chromosome spreads from the imaginal discs. The developing gonads in Ae. albopictus fourth-instar larvae displayed a clear-cut sexual dimorphism that allowed us to sort male and female larval stages. Specifically, the developing testes, located ventrally in the sixth larval 
abdominal segment, display a pyriform shape and appear to be divided into transverse pseudochambers, which represent the spermatic tubes with the germ cells irregularly arranged (Fig. 1a). A short filament, i.e. the suspensorial ligament, is visible at the anterior end. The ovaries are fusiform and are located lateroventrally to the dorsal tracheal trunks in the sixth abdominal segment. They contain irregularly distributed round germ cells (Fig. 1b).

\section{Chromosome 1 homologues display sex-related features}

Metaphase chromosome preparations from male and female larvae of the Rimini strain portray a clear and reproducible karyotype with three pairs of chromosomes which, according to the nomenclature of McDonald \& Rai [34], and by analogy to the Ae. aegypti karyotype [32], were designated as chromosomes 1,2 and 3. Apart from the metacentric chromosome 3 , chromosomes 1 and 2 fall in the sub-metacentric category [11].

DAPI staining of the mitotic chromosome complements from several sexed larvae shows consistent differences in the banding patterns between the chromosome 1 homologues in males and females (Fig. 2). In females, both the smaller arms (p-arm) of the chromosome 1 homologues display a DAPI-negative band which, in males, is present only on one of the homologues. The same sex-related banding pattern on chromosome 1 was obtained with Giemsa C-staining (data not shown). On this basis, we further investigated whether the male-specific chromosome 1 homologue lacking the DAPI-negative band might be related to maleness/male differentiation. Considering that a male determining gene, Nix, has been mapped on chromosome 1 in Ae. aegypti [18], we searched for the

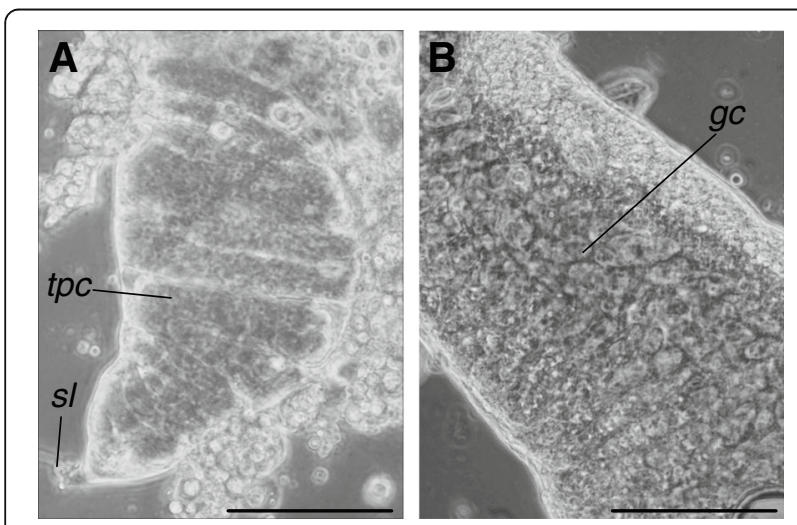

Fig. 1 Gonads of fourth-instar larvae. a Developing testes showing transverse pseudochambers (tpc), which represent the spermatic tubes, and the suspensorial ligament (s). b Developing ovary showing the germ cells (gc). Scale-bars: $100 \mu \mathrm{m}$ presence of a Nix orthologous sequence on this male chromosome in Ae. albopictus.

\section{A Nix orthologous sequence is present in the Ae. albopictus genome}

We used a previously identified 701 bp partial Ae. albopictus Nix cds [18] (GenBank: KP765684.1) as a BLASTN query against the Ae. albopictus genome sequence from the Fellini aka Rimini strain [24]. This resulted in the identification of two contigs (C8454750: 379 bp and C5613352: 257 bp; Additional file 2) that partially overlap the query sequence with $100 \%$ identity at both the 5 and 3 ' ends, extending the Nix sequence by 174 bp and 145 bp at the $5^{\prime}$ and $3^{\prime}$ ends, respectively. The resulting composite sequence of $1020 \mathrm{bp}$ in length (Additional file 3: Figure S1) was then used as a BLASTN query against a transcriptome assembled with Trinity [35] derived from Rimini strain male and female antennae at different physiological states (Gomulski et al., unpublished data). Four transcripts sharing $100 \%$ identity with the Nix sequence (comp292865_c0_seq1: 583bp; comp292865_c0_seq3: 214 bp; comp770372_c0_seq1, 257 bp and comp618873_c0_seq1: 603 bp; Additional file 2) were identified which extended the 3' end of the Nix sequence by $560 \mathrm{bp}$, resulting in a composite sequence of $1580 \mathrm{bp}$ in length (Additional file 3: Figure S1). Finally, this composite sequence was used as a BLASTN query against the Fellini genome, which identified an additional contig (C7387974: 315 bp; Additional file 2) with $100 \%$ identity. As the 1580 bp Nix composite sequence completely encompasses the Fellini genomic contig C7387974 sequence the Nix locus was not extended (Additional file 3: Figure S1). BLASTX analysis of the obtained 1580 Nix sequence against the non-redundant database gave, as expected, a perfect hit to the previously identified partial Ae. albopictus AKI28880.1 NIX protein [18]. In addition, a $171 \mathrm{bp}$ region downstream of that hit also gave $57 / 72 \%$ identity/similarity to the C-terminal part of the $A e$. aegypti NIX protein (AHW46195.1), suggesting that the 1580 bp sequence contains the complete coding sequence. This 1580 bp Nix composite sequence shares $99.9 \%$ identity with a region on a $971 \mathrm{~Kb}$ contig (MNAF02001502.1) present in the Ae. albopictus C6/36 cell line genome [23] (GCA_001876365.2 assembly) and predicted to encode a 282 aa NIX protein. The only difference between the sequences was an 8 bp deletion in the MNAF02001502.1 sequence 56-64 upstream of the Nix start codon.

Examination of our composite sequence indicated that the Ae. albopictus Nix gene consists of two exons of 674 bp and 175 bp separated by a 107 bp intron flanked by donor/acceptor sites. The $5^{\prime}$ donor splice site is $\mathrm{AG}^{\wedge} \mathrm{GUUUGU}$ rather than the canonical $\mathrm{AG}^{\wedge} \mathrm{GUAAGU}$ 

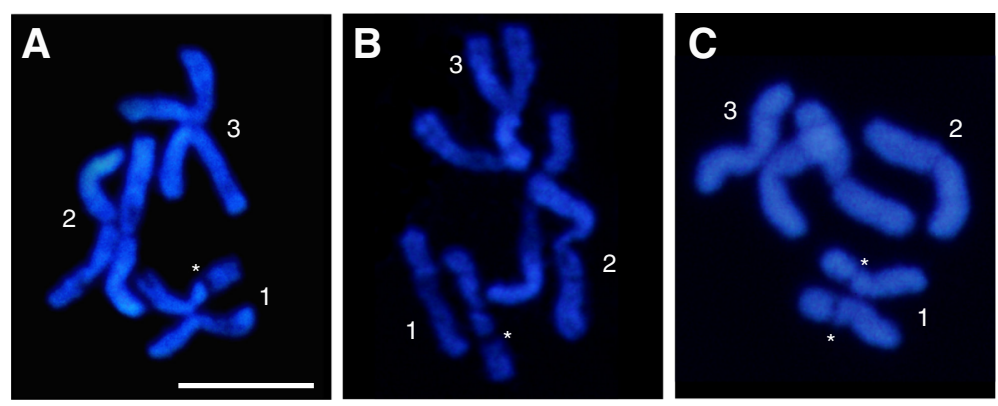

Fig. 2 DAPI stained mitotic chromosomes. a, b Male karyotype showing a DAPI-negative band (asterisk) on only one homologue of chromosome 1. c Female karyotype displaying DAPI-negative bands (asterisks) on both homologues of chromosome 1. Scale-bar: 5 um

sequence for Ae. aegypti [36]. The position of the intron is conserved with respect to Ae. aegypti Nix (NCBI gene report Nix/LOC110678376). The Ae. albopictus Nix gene is predicted to encode a protein of 282 aa with two RNA recognition motifs (RRM smart00360 from 21-63aa and RRM_1 pfam00076 from 205-273aa) (Fig. 3).

\section{Nix is male-linked and is located on the male chromosome} 1 homologue

The use of two primer sets spanning the Nix intron (Nix-309f/r and Nix-833f/r; Fig. 3; Additional file 1: Table S1; Additional file 3: Figure S1) on genomic DNA, derived from male and female adults from different generations of the Rimini strain, produced amplification products only in males (Fig. 4). The sequences of these amplicons were identical to the in-silico sequence with the expected sizes of 309 and $833 \mathrm{bp}$, respectively. The $833 \mathrm{bp}$ sequence includes $602 \mathrm{bp}$ of exon 1, the $107 \mathrm{bp}$ intron, and $124 \mathrm{bp}$ of exon 2, while the 309 bp sequence includes $69 \mathrm{bp}$ of exon 1, the $107 \mathrm{bp}$ intron, and $133 \mathrm{bp}$ of exon 2. Moreover, a 1121 bp sequence of a cloned fragment resulting from amplification of male genomic DNA with a third primer pair spanning the entire coding sequence (Nix-1121f/r; Fig. 3; Additional file 1: Table S1; Additional file 3: Figure S1) showed complete identity with the in-silico sequence and supported the predicted Nix gene structure.

Fluorescence in situ hybridization (FISH) on mitotic chromosomes from male and female sexed larvae, using the 833 Nix fragment as a probe, allowed the localization of Nix to a single position on only one homologous copy of chromosome 1, the male chromosome, i.e. to the homologue that lacks the DAPI-negative band on the p-arm. Specifically, the Nix signal localizes on the q-arm of the male chromosome (Fig. 5a). On the same arm (q) of both homologues of the chromosome 1 pair is located the $18 \mathrm{~S}$ ribosomal gene as demonstrated by FISH using a 757 bp $18 \mathrm{~S}$ rDNA fragment probe (Fig. 5b).

\section{Nix displays very early male transcription during embryogenesis with intron retention}

The same set of primers previously used for Nix DNA amplification (Nix-833f/r and Nix-309f/r) were used for RT-PCR amplification of cDNA derived from single male and female adults. Nix amplicons were consistently obtained only in males (Fig. 6a) and appeared to have the same molecular weight as those obtained from genomic DNA. Cloning and sequencing of Nix-833f/r amplicons confirmed that the Nix transcript was 833 bp in length (like the DNA amplicon) and that it retained the intron sequence. These transcripts are predicted to encode a truncated protein of 233 aa due to the presence of an in frame stop codon within the retained intron sequence (Fig. 3). Nix transcripts were detected in different body compartments including the head, antennae, thorax, abdomen and legs, although the transcript appeared to be more abundant in the head and antennae (Fig. 6b), which is in agreement with Matthews et al. [37] who found highest

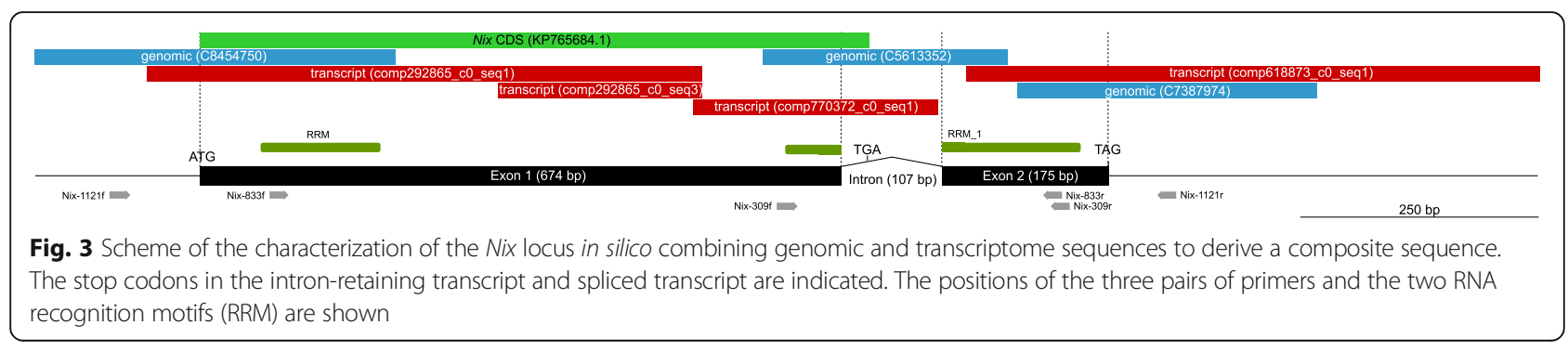




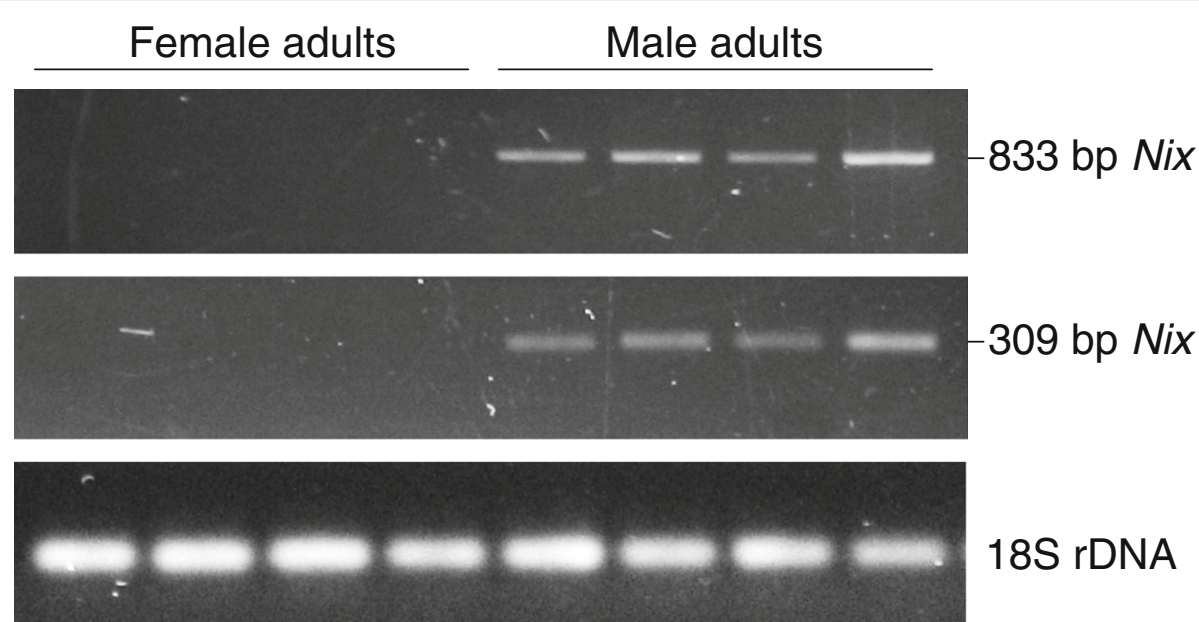

Fig. 4 Amplification of $833 \mathrm{bp}$ and $309 \mathrm{bp}$ Nix fragments from genomic DNA. Amplification of a 185 rDNA fragment in both sexes as a control for DNA integrity

Nix transcript abundances in male brain and antenna tissues of Ae. aegypti. Again, in these body compartments the size of the Ae. albopictus Nix transcript was that obtained from genomic DNA, although in the samples with higher transcript abundances, such as head and antennae, a very faint lower molecular weight band was discernible, corresponding to the putative spliced form. During the different developmental stages, starting from 2-4 $\mathrm{h}$ single embryos, to larvae and pupae (Fig. 7b, d, c, respectively), the Nix male-biased transcription is evident with the two different molecular weight transcripts displaying different relative abundances. This is very evident in 12-24 $h$ embryos where the lower molecular weight fragment appears to be as abundant as the higher molecular weight fragment (Fig. 7a, b). The sequences of the cloned Nix-309f/r RT-PCR amplification products from these 12-24 $\mathrm{h}$ embryos confirmed that these bands represent the 309 bp unspliced intron-retaining Nix transcript fragment and the 202 bp spliced Nix transcript fragment (Additional file 4: Figure S2).

\section{Nix sequences do not display variability in natural populations of Ae. albopictus}

A total of 11 direct Nix-833r/f PCR product sequences were obtained from male mosquitoes collected across the distribution range of Ae. albopictus. Six were from individuals from the home range area: Guangzhou, China, Ban Rai and Chiang Mai in Thailand, while five sequences were derived from two adventive Mediterranean populations, Arco in North Italy and Velika Gorica in Croazia. The partial sequences obtained from these individuals range from 720 bp (Guangzhou) to 767 (Arco) and include the intron sequence. The sequences aligned to the Rimini Nix reference sequence show no polymorphisms (Additional file 5: Figure S3).
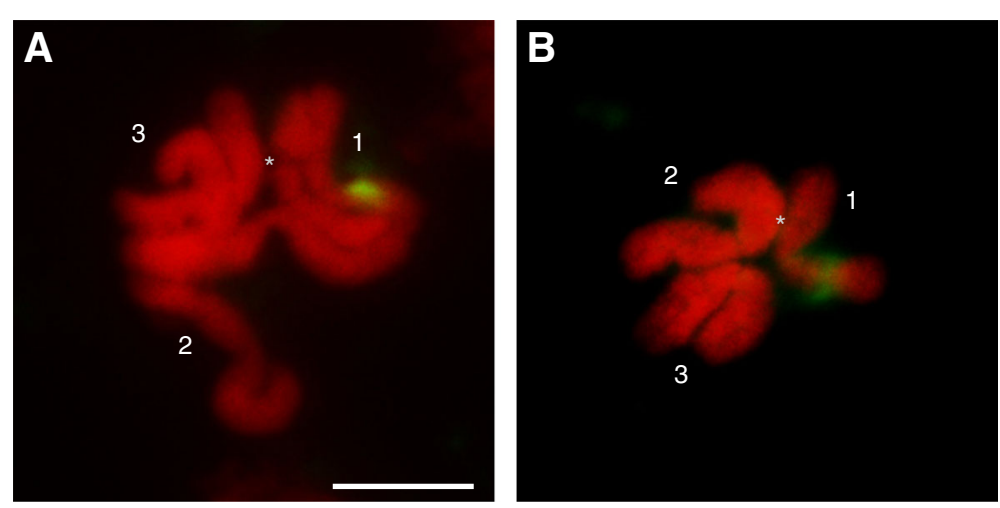

Fig. 5 Fluorescence in situ hybridization (FISH) to mitotic chromosomes using a Nix fragment as probe (a) and a $18 \mathrm{~S}$ rDNA fragment as probe (b). The three pairs of chromosomes are less evident in these images due to somatic pairing [63]. The asterisk indicates the position of the DAPI-negative band on one homologue of chromosome 1. Scale-bar: $5 \mu \mathrm{m}$ 


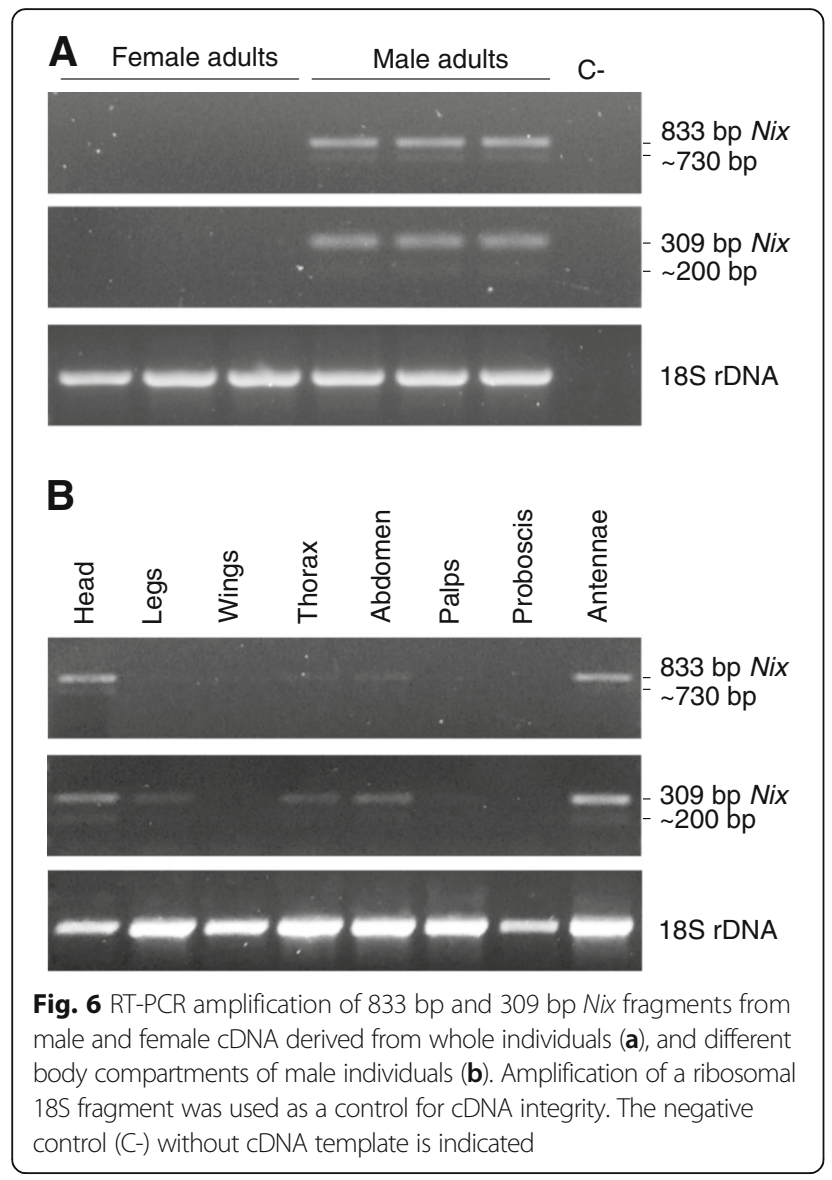

\section{Discussion}

The most important findings of the present study are (i) the identification in Ae. albopictus of the male-specific chromosome 1 homologue, which carries the putative male-determining gene, Nix; (ii) Nix displays precocious and male-specific transcription during development; (iii) Nix displays intron retention within the mature transcript; (iv) the Nix sequence does not appear to be polymorphic in populations across the species range, and (v) markers to sex Ae. albopictus from the single embryo to the pupa have been developed.

\section{The male-specific chromosome 1 homologue contains a potential male determining sequence, Nix}

The protocol that we describe for sexing fourth-instar larvae facilitates the isolation and analysis of the male and female mitotic metaphase chromosome complements. As a result, the male-specific homomorphic chromosome 1 has been recognised through the absence of a DAPI-negative band located about halfway along its p-arm. This band represents a reproducible landmark for this chromosome and for the recognition of the p-arm. This cytological marking system is based on the karyotype present in the long-established reference laboratory strain, Rimini, derived from mosquitoes of an adventive Italian population. The Fellini genome sequence was derived from this strain [24]. Considering the great variation in terms of genome size and repetitive sequences detected in strains from different regions [38-42] we cannot exclude that naturally occurring variation in the distribution and amount of heterochromatin might affect the chromosomal banding pattern, and

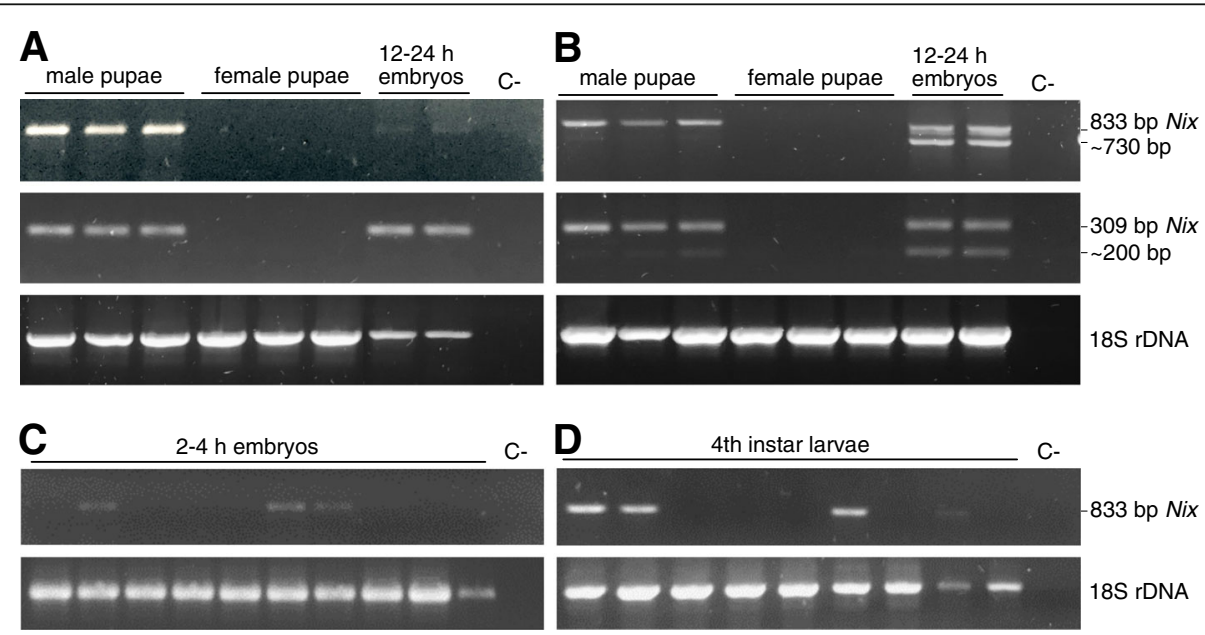

Fig. 7 a DNA amplification of 833 bp and 309 bp Nix fragments from the head/thorax of male and female pupae and from $12-24 \mathrm{~h}$ embryo pools. b RT-PCR amplification of 833 bp and 309 bp Nix fragments from cDNA derived from the abdomens of the same male and female pupae in a, and cDNA from 12-24 h embryo pools. c RT-PCR amplification of 833 bp Nix fragments from cDNA derived from individual embryos processed 2-4 h after deposition. $\mathbf{d}$ RT-PCR amplification of 833 bp Nix fragments from cDNA derived from sexed individual fourth-instar larvae (lanes 1,2,6 and 8 are male larvae). The negative control (C-) without CDNA template is indicated. Amplification of a ribosomal $18 \mathrm{~S}$ fragment was used as a control for DNA/CDNA integrity 
consequently the marking system of the male chromosome 1 in different strains/populations. Such assessment/ evaluation is prevented by the lack of refined chromosome idiograms in this species. Nevertheless, the Giemsa C-banding technique applied to different strains from different origins showed the invariable presence on chromosome 1 of an intercalary band which, in males, is present only on one homologue [16, 43]. Also, in Ae aegypti, Giemsa C-banding demonstrated differences between the male and female chromosome 1 homologues. Aedes aegypti females have a pericentromeric and an intercalary band, both of which may be absent on one of the chromosome 1 homologues in males. However, in this species the banding pattern has been found to be variable in different strains, both for the presence/absence and sizes of the intercalary bands in both males and females $[16,44,45]$. Indeed, physical chromosome-based map analyses demonstrated that the homomorphic chromosome 1 of Ae. aegypti had an evolutionary higher rate of genomic rearrangements than the autosomes, with minisatellites playing the major role in the rapid evolution [17].

Using our cytological marking system as a physical anchor point, we located the male-specific Nix sequence almost halfway along the q-arm of the male-specific chromosome 1 homologue of the Rimini strain. Aedes albopictus Nix, like its Ae. aegypti orthologue, has the attributes to be considered as a male-determining factor [18]. It exhibits persistent male-specific linkage and expression and, more importantly, it displays very early embryonic expression. Indeed, Nix transcription is visible in 2-4 h single embryos. This is the stage that precedes blastoderm formation (at 5-6 $\mathrm{h} \mathrm{[46]),} \mathrm{before} \mathrm{sex} \mathrm{is}$ established. The functional homology between the Ae. albopictus and Ae. aegypti Nix sequences is further supported by their chromosomal locations; they are both located roughly on the same region of the q-arm of chromosome 1 in synteny with the $18 \mathrm{~S}$ ribosomal genes. Synteny conservation and consequently homology between these two chromosomes has been identified using RFLP comparative mapping [47]. Given this background, we can consider the male chromosome 1 carrying Nix as $\mathrm{M}$ and the other copy without it as $\mathrm{m}$, following the terminology of Motara and Rai [16] (Fig. 8). It remains to be assessed whether Ae. albopictus Nix resides in a non-recombining region (sex-determining region, SDR) like Nix in Ae. aegypti [18-20] and the extent of the SDR. This information will provide valuable data on the presence and extent of genetic differentiation between the two chromosome 1 homologues.

\section{Intron retention (IR) in Nix transcripts: a mechanism to modulate gene expression during development?}

Another feature we found in Nix transcription, i.e. intron retention, may act as a mechanism to modulate its

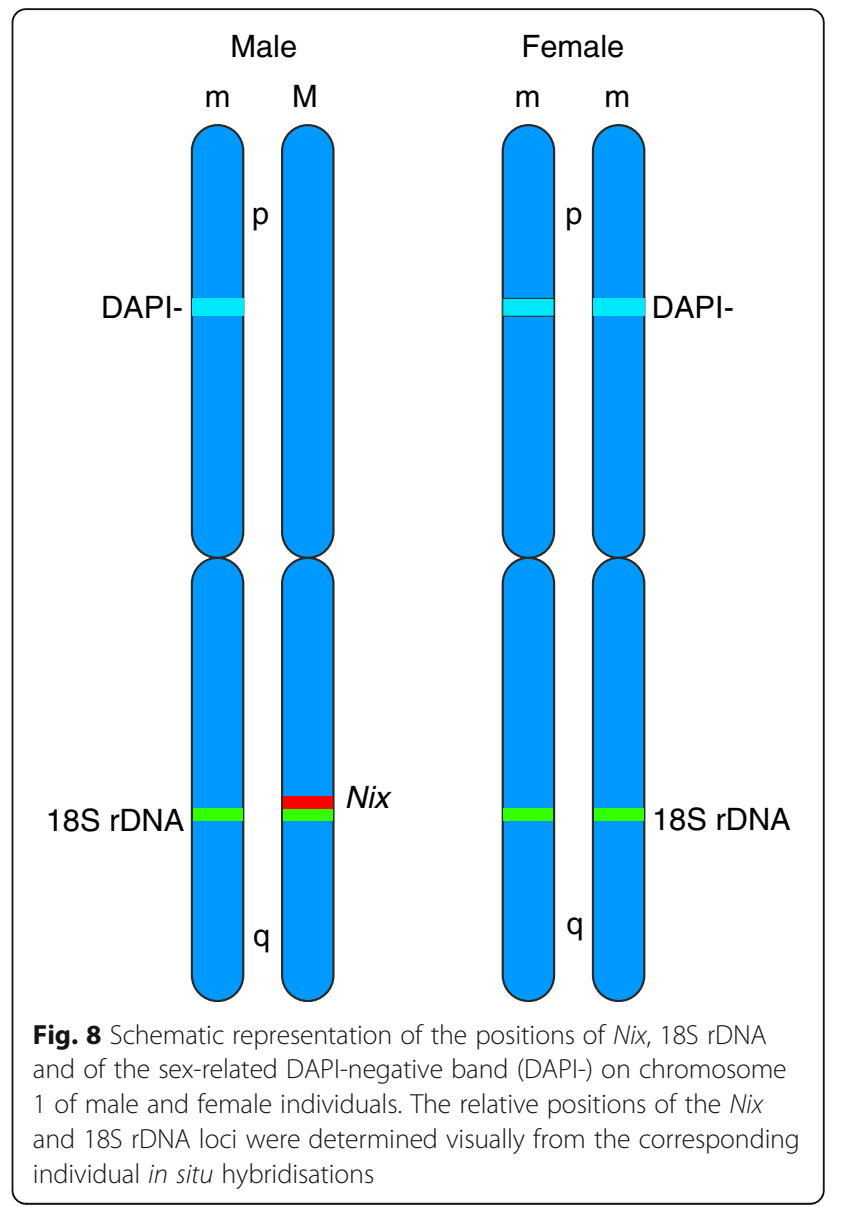

activity. In the different developmental stages, the Nix intron sequence is retained in a variable proportion of transcripts. Intron retention (IR), a type of alternative splicing, is the complete retention of an intron in a mature transcript. Transcripts containing retained introns can have different fates, they may undergo exosome-mediated degradation within the nucleus, or, if they arrive in the cytoplasm, they can give rise to truncated proteins or be degraded by nonsense-mediated decay [48]. IR is likely to be a cellular mechanism to modulate gene expression levels [49-51].

Retained introns tend to be short compared to other introns and have weak $5^{\prime}$ and $3^{\prime}$ splice sites [48]. The Ae. albopictus Nix intron is short at $107 \mathrm{bp}$ and has a non-canonical $5^{\prime}$ donor splice site. The Nix $5^{\prime}$ donor splice site is $\mathrm{AG}^{\wedge} \mathrm{GUUUGU}$ rather than the canonical $\mathrm{AG}^{\wedge} \mathrm{GUAAGU}$ sequence for Ae. aegypti, whereas the Nix $3^{\prime}$ acceptor splice site (UUUCAG^ ${ }^{\wedge} \mathrm{UU}$ ) corresponds to the canonical Ae. aegypti sequence, $\mathrm{UUNCAG}^{\wedge} \mathrm{GU}$ [36]. Retention of the intron in Ae. albopictus Nix may thus be a result of inefficient recognition of the 5 splice site $[48,52]$. That the efficiency of splicing of the intron appears to vary in different body compartments and in different developmental stages is interesting, as intron 
retention has been shown to be more common in transcripts that are less required for the physiology of a particular tissue [53]. Of all the developmental stages that we analysed, the most evident presence of the spliced Nix isoform was in the 12-24 h embryos, where the spliced and intron-retention forms appeared to be almost equally abundant (Fig. 7b). This developmental range, in Ae. aegypti, covers a very rapid embryonic segmentation kinetics which includes the onset of gastrulation $(12 \mathrm{~h})$, initiation of segmentation and ventral cord formation (15 h), labral lobe formation (18 h) and anterior and posterior midgut invagination formation (19 h) [46]. The fainter bands, corresponding to the spliced isoform, visible in male pupae (Fig. 7b), whole adult males (Fig. 6a) and some adult male body compartments especially the head and antennae (Fig. $6 \mathrm{~b}$ ), suggest that some splicing is occurring throughout the tissues or, at least, in a subset of cells.

The Ae. albopictus NIX protein has a moderate, partial identity (39\%) with D. melanogaster Transformer 2 protein (TRA2), one of the key genes in the sex-determination pathway. TRA2 inhibits the splicing of the M1 intron of its own tra2 pre-mRNA. The resulting transcripts encode a non-functional protein that lacks one of the two regions rich in arginine and serine (RS domains) that facilitate protein-protein interactions critical in the regulation of alternative splicing. These M1 intron-retaining tra2 RNAs are found almost exclusively in the male germline where they constitute $50 \%$ of tra 2 mRNA. M1 retention has been shown to be a negative-feedback mechanism by which the functional TRA2 limits its own synthesis by promoting the accumulation of M1-containing transcripts. The absence of this negative-feedback mechanism in the Drosophila male germ line results in defective spermatogenesis $[49,54]$.

In Ae. albopictus, the retention of the intron in the mature Nix cDNA is predicted to result in a truncated protein of 233 aa due to the presence of a stop codon within the intron sequence. Just as the Drosophila truncated TRA2 proteins encoded by the IR transcripts lack an RS domain, the truncated Ae. albopictus IR NIX proteins lack one of the two RRMs. Like TRA2, NIX in $A e$. aegypti, has been hypothesized to be a splicing factor that is responsible, directly or indirectly, for the male-specific splicing of $d s x$ and fru [22]. This male-specific splicing of these two genes, which are important components of the sex-determination pathways of many insects, triggers cascades that give rise to the development of sexually dimorphic traits [55]. Indeed, silencing of Nix results in female splice variants of $d s x$ and $f r u$ in male Ae. aegypti and morphological feminization, and ectopic expression of Nix in female embryos results in masculinization of the resulting adults [18]. The roles of the multiple tra-2 homologues in Aedes mosquitoes are not clear. Knockdown of three of the four tra-2 paralogues in Ae. aegypti did not alter $d s x$ splicing or cause sex conversion of the resulting adults [55]. One of the tra-2 paralogues has however been shown to be implicated in sex ratio distortion in $A e$. aegypti apparently due to segregation distortion that affects gametic function, and female-specific zygotic lethality [56].

\section{The Nix sequence is invariable in populations}

Despite the high degree of genetic variability present in native Asian Ae. albopictus populations and maintained in adventive populations [4,57], the Nix sequence, covering most of the two exons and including the short intron (720-767 bp), shows no nucleotide variability in the populations analysed. Nix has a very short intron and encodes a small protein: this condition is expected considering its putative male-determining function and its very early zygotic expression [46]. In Drosophila, the majority of early zygotic expressed genes are intronless or have small introns and encode small proteins $[58,59]$. Nix transcription in Ae. albopictus has been detected before the syncytial blastoderm stage, where several waves of rapid mitotic cell division occur and when the transcription of zygotic genes begins. During these rapid mitotic cycles the time required to transcribe genes with long primary transcripts is not trivial and poses constrains in terms of cost to their expression $[58,60]$. Indeed, in Drosophila long zygotically expressed genes show delayed expression compared to shorter ones. This delay results from the inability to completely transcribe their long transcripts. It has been suggested that highly expressed early zygotic genes are subject to purifying selection to maintain short transcript length [61]. Selection may be acting in limiting the expansion of the Ae. albopictus Nix intron to preserve efficient transcription in the embryo. A similar lack of polymorphism was reported in the Y-linked gene Dhc-Yh3 in lines of D. melanogaster and $D$. simulans [62]. Numerous factors can help interpret the lack of variation of these genes, such as the absence of recombination, codon bias and selective sweeps.

\section{Conclusions}

Here we provide basic information essential to improve existing technologies, involving the rearing and release of large numbers of Ae. albopictus mosquitoes, to eliminate or modify its populations. The characterization of the male and female mitotic karyotypes and the identification of the male-specific chromosome 1 homologue that carries the putative male-determining gene, Nix, are essential prerequisites for the development of any genetic sexing system. They are a premise for the development of male and female physical maps and genome 
analyses in Ae. albopictus. This will facilitate the identification of genomic traits controlling sex determination/ differentiation, reproduction, development, physiology and those related to susceptibility/refractoriness to different pathogens. The characterisation of the Nix gene in Ae. albopictus represents an initial step in the analysis of the sex determination cascade in this species: we have identified a possible mechanism that regulates its expression in embryogenesis and during development. This provides insights for developing new genetic strategies that convert female mosquitoes into harmless males. Finally, using morphological, cytogenetics and molecular approaches we have developed markers which are useful for sexing pre-imaginal Ae. albopictus individuals from the embryo to pupal stage.

\section{Additional files}

\section{Additional file 1: Table S1. Primer sequences. (DOCX $12 \mathrm{~kb}$ )}

Additional file 2: Text. Genomic (Fellini aka Rimini) and transcriptome (Rimini) sequences used in the assembly of the Ae. albopictus Nix locus. (TXT $2 \mathrm{~kb}$ )

Additional file 3: Figure S1. (PDF) Sequence of the Nix locus showing the positions of the exons (green box), intron and predicted amino acid sequence. The positions of the primers are shown. (PDF $76 \mathrm{~kb}$ )

Additional file 4: Figure S2. (PDF) Alignment of the 12-24 h embryo-derived Nix transcript sequences showing a transcript with intron retention (unspliced) and a spliced transcript. The predicted translation products of the fragments are shown. (PDF $35 \mathrm{~kb}$ )

Additional file 5: Figure S3. (PDF) Alignment of the $833 \mathrm{bp}$ Nix fragment from the Rimini reference sequence with the 10 Nix-833r/f PCR product sequences from individuals from four wild population samples (Guangzhou, Arco, Ban Rai, and Velika Gorica) and one sequence from the Chiang Mai pool. Dashes represent missing data and dots indicate identity to the reference sequence. (PDF $144 \mathrm{~kb}$ )

\section{Acknowledgements}

This study was performed within the Food and Agriculture Organization/ International Atomic Energy Agency (FAO/IAEA) research Coordinated Research Project "Exploring genetic, molecular, mechanical and behavioural methods of sex separation in mosquitoes" and benefited from discussions at IAEA funded meetings therein. It is also operating within the framework of the University of Pavia Strategic Theme "Towards a governance model for international migration: an interdisciplinary and diachronic perspective" (MIGRAT-IN-G), and in the framework of the Agreement for "The International Joint Laboratory on Vector-Borne Diseases" established between Southern Medical University, China, and University of Pavia, Italy. The research was performed within the Dept. of Biology and Biotechnology 'L. Spallanzani' recognised within the "Dipartimenti di Eccellenza Program (2018-2022)" by the Italian Ministry of Education, University and Research (MIUR).The authors thank the editor and the two referees for their constructive comments.

\section{Funding}

This work was partially supported by the Crowdfunding Initiative "Universitiamo: Fight the mosquito bite", University of Pavia. Publication costs for this study was provided by the International Atomic Energy Agency as part of the Coordinated Research Project "Exploring genetic, molecular, mechanical and behavioural methods of sex separation in mosquitoes".

\section{Availability of data and materials}

The datasets supporting the conclusions of this article are included within the article and its additional files.

\section{About this supplement}

This article has been published as part of Parasites \& Vectors Volume 11 Supplement 2, 2018: Exploring genetic molecular, mechanical and behavioural methods of sex separation in mosquitoes. The full contents of the supplement are available online at https://parasitesandvectors.biomedcentral.com/ articles/supplements/volume-11-supplement-2.

\section{Authors' contributions}

ARM, LMG, GG and FS conceptualized and supervised the study; validated, analysed and interpreted the data. ARM and GG acquired funding, samples and administered the project. MM, FS, LMG, ADC, MM and GS devised the methodology and performed the experiments. LMG visualized the results of the study. ARM, LMG, FS and GG wrote the daft of the paper. All authors read and approved the final manuscript.

Ethics approval and consent to participate

The insect samples were received in accordance with European Union rules (Directive 97/78/CE)

\section{Consent for publication}

Not applicable.

\section{Competing interests}

The authors declare that they have no competing interests.

\section{Publisher's Note}

Springer Nature remains neutral with regard to jurisdictional claims in published maps and institutional affiliations.

\section{Author details}

'Department of Biology and Biotechnology "L. Spallanzani", University of Pavia, Pavia, Italy. 'Department of Genetic Medicine and Development, University of Geneva Medical School, and Swiss Institute of Bioinformatics, Geneva, Switzerland.

\section{Published: 24 December 2018}

\section{References}

1. Paupy C, Delatte H, Bagny L, Corbel V, Fontenille D. Aedes albopictus, an arbovirus vector: from the darkness to the light. Microbes Inf. 2009;11:1177-85.

2. Jupille $H$, Seixas G, Mousson L, Sousa CA, Failloux AB. Zika virus, a new threat for Europe? PLoS Negl Trop Dis. 2016;10:e0004901.

3. Bonizzoni M, Gasperi $G$, Chen $X$, James $A A$. The invasive mosquito species Aedes albopictus: current knowledge and future perspectives. Trends Parasitol. 2013;29(9):460-8.

4. Manni M, Guglielmino CR, Scolari F, Vega-Rúa A, Failloux AB, Somboon P, et al. Genetic evidence for a worldwide chaotic dispersion pattern of the arbovirus vector, Aedes albopictus. PLoS Negl Trop Dis. 2017;11:e0005332.

5. Kotsakiozi P, Richardson JB, Pichler V, Favia G, Martins AJ, Urbanelli S, et al. Population genomics of the Asian tiger mosquito, Aedes albopictus: insights into the recent worldwide invasion. Ecol Evol. 2017;7:10143-57.

6. Rezza G, Nicoletti L, Angelini R, Romi R, Finarelli AC, Panning M, et al. Infection with chikungunya virus in Italy: an outbreak in a temperate region. Lancet. 2007;370:1840-6.

7. Venturi G, Di Luca M, Fortuna C, Remoli ME, Riccardo F, Severini F, et al. Detection of a chikungunya outbreak in central Italy, August to September 2017. Euro Surveill. 2017;22:17-00646.

8. Delisle E, Rousseau C, Broche B, Leparc-Goffart I, L'Ambert G, Cochet A, et al. Chikungunya outbreak in Montpellier, France, September to October 2014. Euro Surveill. 2015;20:21108.

9. Lees RS, Gilles JR, Hendrichs J, Vreysen MJ, Bourtzis K. Back to the future: the sterile insect technique against mosquito disease vectors. Curr Opin Insect Sci. 2015;10:156-62.

10. Gilles JR, Schetelig MF, Scolari F, Marec F, Capurro ML, Franz G, et al. Towards mosquito sterile insect technique programmes: exploring genetic, molecular, mechanical and behavioural methods of sex separation in mosquitoes. Acta Trop. 2014;132(Suppl):S178-87.

11. Sallam FAE, Abou El Ela RG. Cytogenetic analysis of metaphase chromosomes from pupal testes of four mosquito species using fluorescence in situ hybridization technique (FISH). World J Microbiol Biotechnol. 2005;21:4. 
12. Rai KS. A comparative study of mosquito karyotypes. Ann Entomol Soc Amer. 1963;56:11.

13. Rai KS, Black WC. Mosquito genomes: structure, organization, and evolution. Adv Genet. 1999:41:1-33.

14. McClelland GAH. Sex-linkage in Aedes aegypti. Trans Roy Soc Trop Med Hyg. 1962:56:4.

15. Toups MA, Hahn MW. Retrogenes reveal the direction of sex-chromosome evolution in mosquitoes. Genetics. 2010;186:763-6.

16. Motara MA, Rai KS. Giemsa C-banding patterns in Aedes (Stegomyia) mosquitoes. Chromosoma. 1978;70:8.

17. Timoshevskiy VA, Kinney NA, deBruyn BS, Mao C, Tu Z, Severson DW, et al. Genomic composition and evolution of Aedes aegypti chromosomes revealed by the analysis of physically mapped supercontigs. BMC Biol. 2014;12:27.

18. Hall AB, Basu S, Jiang X, Qi Y, Timoshevskiy VA, Biedler JK, et al. A male-determining factor in the mosquito Aedes aegypti. Science. 2015; 348:1268-70.

19. Hall AB, Timoshevskiy VA, Sharakhova MV, Jiang X, Basu S, Anderson MA, et al. Insights into the preservation of the homomorphic sex-determining chromosome of Aedes aegypti from the discovery of a male-biased gene tightly linked to the M-locus. Genome Biol Evol. 2014;6:179-91.

20. Fontaine A, Filipovic I, Fansiri T, Hoffmann AA, Cheng C, Kirkpatrick M, et al. Extensive genetic differentiation between homomorphic sex chromosomes in the mosquito vector, Aedes aegypti. Genome Biol Evol. 2017;9:2322-35.

21. Salz HK, Erickson JW. Sex determination in Drosophila: The view from the top. Fly (Austin). 2010;4:60-70.

22. Adelman ZN, Tu Z. Control of mosquito-borne infectious diseases: sex and gene drive. Trends Parasitol. 2016;32:219-29.

23. Miller JR, Koren S, Dilley KA, Puri V, Brown DM, Harkins DM, et al. Analysis of the Aedes albopictus C6/36 genome provides insight into cell line utility for viral propagation. Gigascience. 2018;7:1-13.

24. Dritsou V, Topalis P, Windbichler N, Simoni A, Hall A, Lawson D, et al. A draft genome sequence of an invasive mosquito: an Italian Aedes albopictus. Pathog Glob Health. 2015;109:207-20.

25. Moorefield HH. Sexual dimorphism in mosquito pupae. Mosquito News. 1951;11:3.

26. Vargas MV. Sexual dimorphism of larvae and pupae of Aedes aegypti (Linn.). Mosquito News. 1968;28:6.

27. Baruffi L, Damiani G, Guglielmino CR, Bandi C, Malacrida AR, Gasperi G. Polymorphism within and between populations of Ceratitis capitata: comparison between RAPD and multilocus enzyme electrophoresis data. Heredity (Edinb). 1995;74:425-37.

28. Untergasser A, Cutcutache I, Koressaar T, Ye J, Faircloth BC, Remm M, et al. Primer3 - new capabilities and interfaces. Nucleic Acids Res. 2012:40:e115.

29. Altschul SF, Gish W, Miller W, Myers EW, Lipman DJ. Basic local alignment search tool. J Mol Biol. 1990;215:403-10.

30. Camacho C, Coulouris G, Avagyan V, Ma N, Papadopoulos J, Bealer K, et al. BLAST+: architecture and applications. BMC Bioinformatics. 2009;10:421.

31. Marchler-Bauer A, Derbyshire MK, Gonzales NR, Lu S, Chitsaz F, Geer LY, et al. CDD: NCBl's conserved domain database. Nucleic Acids Res. 2015; 43(Database issue):D222-6.

32. Sharakhova MV, Timoshevskiy VA, Yang F, Demin S, Severson DW, Sharakhov IV. Imaginal discs - a new source of chromosomes for genome mapping of the yellow fever mosquito Aedes aegypti. PLoS Negl Trop Dis. 2011;5:e1335.

33. Pimpinelli $S$, Berloco $M$, Fanti $L$, Dimitri $P$, Bonaccorsi $S$, Marchetti $E$, et al. Transposable elements are stable structural components of Drosophila melanogaster heterochromatin. Proc Natl Acad Sci USA. 1995;92:3804-8.

34. McDonald PT, Rai KS. Correlation of linkage groups with chromosomes in the mosquito, Aedes aegypti. Genetics. 1970;66:475-85.

35. Grabherr MG, Haas BJ, Yassour M, Levin JZ, Thompson DA, Amit I, et al. Fulllength transcriptome assembly from RNA-Seq data without a reference genome. Nat Biotechnol. 2011;29:644-52.

36. Nguyen $\mathrm{H}$, Das $U$, Wang $B$, Xie J. The matrices and constraints of GT/AG splice sites of more than 1000 species/lineages. Gene. 2018;600:10.

37. Matthews BJ, McBride CS, DeGennaro M, Despo O, Vosshall LB. The neurotranscriptome of the Aedes aegypti mosquito. BMC Genomics. 2016;17:32.

38. Rao PN, Rai K. Inter and intraspecific variation in nuclear DNA content in Aedes mosquitoes. Heredity (Edinb). 1987;59:253-8.
39. McLain DK, Rai KS, Fraser MJ. Intraspecific and interspecific variation in the sequence and abundance of highly repeated DNA among mosquitoes of the Aedes albopictus subgroup. Heredity (Edinb). 1987;58:373-81.

40. Black WC, Rai KS. Genome evolution in mosquitoes: intraspecific and interspecific variation in repetitive DNA amounts and organization. Genet Res. 1988;51:185-96.

41. Black WC, MCLain DK, Rai KS. Patterns of variation in the rDNA cistron within and among world populations of a mosquito, Aedes albopictus (Skuse). Genetics. 1989:121:539-50.

42. Waterhouse RM, Chen XG, Bonizzoni M, Gasperi G, Participants IWAA. The third International Workshop on Aedes albopictus: building scientific alliances in the fight against the globally invasive Asian tiger mosquito. Pathogens and Global Health. 2017;111:161-5.

43. Newton ME, Southern DI, Wood RJ. X and Y chromosomes of Aedes aegypti (L.) distinguished by Giemsa C-banding. Chromosoma. 1974:49:41-9.

44. Wallace AJ, Newton ME. Heterochromatin diversity and cyclic responses to selective silver staining in Aedes aegypti (L.). Chromosoma. 1987;95:89-93.

45. Sousa RC, Bicudo HEMC. Heterochromatic banding pattern in two Brazilian populations of Aedes aegypti. Genetica. 1999;105:93-9.

46. Raminani LN, Cupp EW. Embryology of Aedes aegypti (L.) (Dipter: Culicidae): organogenesis. J Insect Morphol Embryol. 1978;7:25.

47. Severson DW, Thathy V, Mori A, Zhang Y, Christensen BM. Restriction fragment length polymorphism mapping of quantitative trait loci for malaria parasite susceptibility in the mosquito Aedes aegypti. Genetics. 1995;139:1711-7.

48. Sibley CR, Blazquez L, Ule J. Lessons from non-canonical splicing. Nat Rev Genet. 2016;17:407-21

49. Chandler DS, Qi J, Mattox W. Direct repression of splicing by transformer-2. Mol Cell Biol. 2003;23:5174-85.

50. Bourdin CM, Moignot B, Wang L, Murillo L, Juchaux M, Quinchard S, et al. Intron retention in mRNA encoding ancillary subunit of insect voltage-gated sodium channel modulates channel expression, gating regulation and drug sensitivity. PLoS One. 2013;8:e67290.

51. Wang M, Branco AT, Lemos B. The $Y$ chromosome modulates splicing and sex-biased intron retention rates in Drosophila. Genetics. 2018;208:1057-67.

52. Sakabe NJ, de Souza SJ. Sequence features responsible for intron retention in human. BMC Genomics. 2007:8:59.

53. Braunschweig U, Barbosa-Morais NL, Pan Q, Nachman EN, Alipanahi B, Gonatopoulos-Pournatzis T, et al. Widespread intron retention in mammals functionally tunes transcriptomes. Genome Res. 2014;24:1774-86.

54. McGuffin ME, Chandler D, Somaiya D, Dauwalder B, Mattox W. Autoregulation of transformer-2 alternative splicing is necessary for normal male fertility in Drosophila. Genetics. 1998;149:1477-86.

55. Salvemini M, D'Amato R, Petrella V, Aceto S, Nimmo D, Neira M, et al. The orthologue of the fruitfly sex behaviour gene fruitless in the mosquito Aedes aegypti: evolution of genomic organisation and alternative splicing. PLoS One. 2013;8:e48554.

56. Hoang KP, Teo TM, Ho TX, Le VS. Mechanisms of sex determination and transmission ratio distortion in Aedes aegypti. Parasit Vectors. 2016;9:49.

57. Manni M, Gomulski LM, Aketarawong N, Tait G, Scolari F, Somboon P, et al. Molecular markers for analyses of intraspecific genetic diversity in the Asian tiger mosquito, Aedes albopictus. Parasit Vectors. 2015;8:188.

58. Guilgur LG, Prudêncio P, Sobral D, Liszekova D, Rosa A, Martinho RG. Requirement for highly efficient pre-mRNA splicing during Drosophila early embryonic development. Elife. 2014;3:e02181.

59. Martinho RG, Guilgur LG, Prudêncio P. How gene expression in fast-proliferating cells keeps pace. Bioessays. 2015;37:514-24.

60. Gubb D. Intron-delay and the precision of expression of homeotic geneproducts in Drosophila. Developm Genetics. 1986;7:119-31.

61. Artieri CG, Fraser HB. Transcript length mediates developmental timing of gene expression across Drosophila. Mol Biol Evol. 2014;31:2879-89.

62. Zurovcova M, Eanes WF. Lack of nucleotide polymorphism in the Y-linked sperm flagellar dynein gene Dhc-Yh3 of Drosophila melanogaster and D. simulans. Genetics. 1999;153:1709-15.

63. Wandall A, Svendsen A. Transition from somatic to meiotic pairing and progressional changes of the synaptonemal complex in spermatocytes of Aedes aegypti. Chromosoma. 1985;92:254-64. 